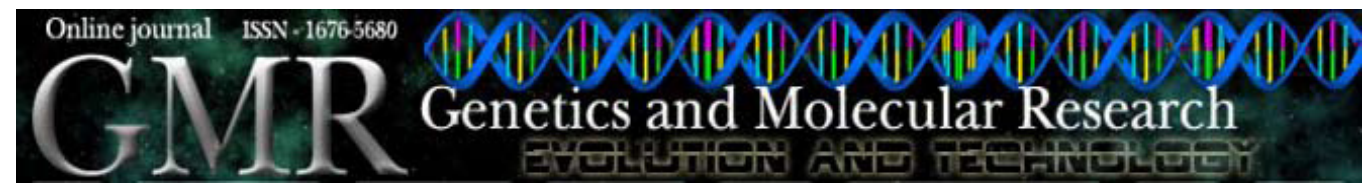

\title{
Evolution of the SEC1 gene in New World monkey lineages (Primates, Platyrrhini)
}

\author{
B.N. Borges ${ }^{1}$, T.S. Paiva ${ }^{2}$ and M.L. Harada ${ }^{1}$ \\ 'Laboratório de Biologia Molecular "Francisco Mauro Salzano", \\ Instituto de Ciências Biológicas, Universidade Federal do Pará, \\ Cidade Universitária Prof. José da Silveira Netto, Belém, PA, Brasil \\ ${ }^{2}$ Departamento de Zoologia, Laboratório de Protistologia, \\ Instituto de Biologia, CCS, Universidade Federal do Rio de Janeiro, \\ Ilha do Fundão, Rio de Janeiro, RJ, Brasil \\ Corresponding author: B.N. Borges \\ E-mail: bnborges@ufpa.br
}

Genet. Mol. Res. 7 (3): 663-678 (2008)

Received May 20, 2008

Accepted June 20, 2008

Published July 29, 2008

ABSTRACT. The structure and evolution of the SEC1 gene were examined for the first time in New World primates of the genera Alouatta, Aotus, Ateles, Brachyteles, Callicebus, Callithrix, Cebus, Chiropotes, Lagothrix, Leontopithecus, Pithecia, Saguinus, and Saimiri. This gene has a high CG content (63.8\%) and an estimated heterogeneous size ranging from 795 (Callithrix) to 1041 bp (Pithecia), due to numerous indel events. Similar to other fucosyltransferases, three conserved regions are shared by these primates, except for the callitrichines, Aotus and Pithecia, in which indel events resulted in premature stop codons that are related to the production of a supposedly non-functional protein. Phylogenetic analysis of the SEC1 gene, transition/transversion rates, and nucleotide sequence alignment support the hypothesis that primate SEC1 evolved by divergent evolution, and that the lack of activity in some lineages occurred independently at least twice in New World primates, once in the Aotus-Cebus-Callitrichinae group and again in Pithecia. Likelihood-based inference of ancestral states for the activity of SEC1 leads us to suppose that inactivation of SEC1 in the Callitrichinae was a result of a more complex series of events than in Pithecia.

Key words: Fucosyltransferases; Divergent evolution; Molecular inactivation; New World monkeys 


\section{INTRODUCTION}

The $\alpha 1,2$ fucosyltransferases ( $\alpha 1,2$ FUT) are a family of similarly structured type II transmembrane proteins that have a short $\mathrm{NH}_{2}$-terminal cytoplasmic tail, a signal membrane anchor domain, a stem region and a globular $\mathrm{COOH}$-terminal catalytic domain within the luminal trans-Golgi compartment (Paulson and Colley, 1989). They are coded by FUT1 $(\mathrm{H})$, FUT2 (SE) and SEC1 genes, which are grouped in the long arm of human chromosome 19 (19q 13.3) (Reguigne-Arnould et al., 1995, 1996).

Although differing in genomic organization and specificities of encoding enzymes, all $\alpha 1,2$ FUT share some characteristics, including i) a monoexonic coding sequence, ii) a complex tissue- and cell type-specific expression pattern regulated by several 5' untranslated exons, and iii) encode an enzyme that catalyses the final step in fucosylated glycan synthesis (Costache et al., 1997; Saunier et al., 2001; Javaud et al., 2003).

In humans, tissue-specific expression patterns of FUT1 and FUT2 genes are well known; FUT1 is expressed in vascular endothelium and erythrocytes, while FUT2 is expressed in epithelial cells and body fluids, such as saliva. Conversely, SEC1 is inactive due to the deletion of two nucleotides, resulting in a truncate polypeptide with 246 amino acids. This gene also presents some characteristics of a non-autonomous retrotransposon, such as association with Alu-like elements and presence of a poly-A tail (Kelly et al., 1995; Saunier et al., 2001). On the other hand, Hitoshi et al. (1995) and Barreaud et al. (2000) showed that these three genes are fully activated in bovines and rabbits.

SEC1 was investigated in non-human primates by Apoil et al. (2000). They proposed that it is inactive in chimpanzees, gorillas and marmosets, due to nonsense mutations in apes and several deletion events in marmosets. They also suggested that the SEC1 gene is active in other Neotropical primate genera (e.g., Saimiri), due to the absence of known mutations and indel events that could cause a premature stop codon (Hitoshi et al., 1995, 1996; Apoil et al., 2000).

We compared SEC1 DNA sequences from 13 New World monkey genera and analyzed their evolution in primates.

\section{MATERIAL AND METHODS}

\section{Isolation, amplification and DNA sequencing}

DNA was obtained from 19 samples of 13 New World monkey genera (from the primate blood sample bank of Laboratório de Biologia Molecular, Universidade Federal do Pará) with the phenolic extraction protocol (Sambrook et al., 1989). The coding region was amplified using the primers designed by Apoil et al. (2000) specifically for New World monkeys. The polymerase chain reaction contained $1 \mathrm{X}$ reaction buffer, $100 \mathrm{ng}$ DNA, 0.4 $\mathrm{mM}$ of each primer, $0.03 \mathrm{U} / \mu \mathrm{L}$ Taq DNA polymerase, $1.4 \mathrm{mM} \mathrm{MgCl}, 0.1 \mu \mathrm{g} / \mu \mathrm{L}$ BSA and $10 \mathrm{mM}$ of each dNTP. The following conditions were applied (repeated 35 times): $94^{\circ} \mathrm{C}$ for $50 \mathrm{~s}, 60^{\circ} \mathrm{C}$ for $50 \mathrm{~s}$ and $72^{\circ} \mathrm{C}$ for $1.5 \mathrm{~min}$. Amplified fragments were purified with the Wizard ${ }^{\circledR}$ PCR Preps kit (Promega), and sequenced by the dideoxyterminal method (Sanger et al., 1977), using an ABI 377 (Applied Biosystems) automatic sequencer. 


\section{Databases and sequence alignment}

The nucleotide database was composed of the sequences obtained as described above (Table 1), with addition of others from the literature (GenBank accession codes within parentheses): Callithrix jacchus (AF111938), Gorilla gorilla (AB006611), Homo sapiens (U17895), Hylobates agilis (AB006609), Macaca fascicularis (AF112475), Macaca mulatta (AF080608), Pan troglodytes (AB006612), Pongo pygmaeus (AB006610), and Saimiri sciureus ssp (AF111937).

Sequences from Mus musculus (NM019934), Bos taurus (AF187851) and Oryctolagus cuniculus (X80225) were included in the amino acid database to compare their functional proteins with the protein sequences obtained from New World monkeys. Both nucleotide and amino acid sequences were aligned automatically by the software BioEdit 5.0.9 (Hall, 1999), using the default options, and then refined manually. Mutational saturation (transitions and transversions over divergence) was tested with the software DAMBE 4.2.13 (Xia and Xie, 2001), using Kimura's distance (Kimura, 1980), and no saturation was observed in these New World monkeys.

\section{Evolutionary analyses}

Transition and transversion rates among nucleotides, relative nucleotide and amino acid frequencies, codon usage, number of synonymous $(\mathrm{dS})$ and nonsynonymous $(\mathrm{dN})$ substitutions per site were calculated by the modified Nei-Gojobori method (Zhang et al., 1998) and computed using MEGA 3.0 (Kumar et al., 2004). Positive selection was assessed by testing the hypothesis $\mathrm{H}_{1}$ : $\mathrm{dN}>\mathrm{dS}$ against a null hypothesis $\mathrm{H}_{0}$ : $\mathrm{dN}=\mathrm{dS}$ using the one-tailed Z-test for selection (Nei and Kumar, 2000), implemented in MEGA; the standard error was calculated by the bootstrap method, using 1000 replicates; $\mathrm{P}<0.05$ was considered to be statistically significant.

The evolutionary rate of the gene (r) was calculated as $\mathrm{K} / 2 \mathrm{~T}$, where $\mathrm{K}$ is the distance between the lineages analyzed and $\mathrm{T}$ is their divergence time ( $\mathrm{Li}, 1997)$. To check if all sequences evolved at similar rates, a relative rate test was performed with the software HyPhy (Pond et al., 2005) using HKY85 (Hasegawa et al., 1985) model parameters.

Phylogenetic analysis was performed using the software PAUP*4b10 (Swofford, 2003), with parsimony criterion and application of successive weighting (Farris, 1969). Gaps were coded as a "fifth base" in order to preserve their information. Initially, 16 fundamental most-parsimonious trees were obtained through the parsimony-ratchet search strategy (Nixon, 1999), implemented via the accessory software PaupRat (Sikes and Lewis, 2001), using the "tree bisection and reconnection" branch-swapping algorithm and 200 ratchet iterations. Characters were then re-weighted based on their rescaled consistency index, and heuristic searches (iterations) were performed using the "tree bisection and reconnection" with 300 replicates of random sequence addition until the weights stabilized for two consecutive runs (Kitching et al., 1998). The analysis resulted in a single most parsimonious tree (Figure 1). The root was placed a posteriori following Nixon and Carpenter (1993), assuming Gorilla, Homo, Hylobates, Macaca, Pan, and Pongo as outgroups. Nodal support was assessed by 1000 bootstrap replicates (Felsenstein, 1985). 
Divergence times among the sequences were estimated in order to date the split events within the New World monkey SEC1 lineages. First, model parameters of unequal nucleotide substitution, transition/transversion rate and heterogeneity rate among sites were obtained with the software BASEML, included in PAML 3.15 (Yang, 1997), using the likelihood criterion, following Rutschmann (2005). Next, branch lengths for the phylogenetic tree were estimated with the software ESTBRANCHES from the MULTIDISTRIBUTE package (see Thorne et al., 1998); these were used for Bayesian molecular clock inferences (Thorne et al., 1998; Kishino et al., 2001; Thorne and Kishino, 2002), performed with the MULTDIVTIME software, also included in aforementioned package. The Markov Chain Monte Carlo was run for 1,000,000 cycles before taking the first sample, and then sampled 10,000 times with 100-cycle intervals.

Time constraints were specified for three tree nodes, corresponding to data from Opazo et al. (2006). The nodes represent the Cebidae divergence (20.2-22.8 million years ago, MYA), the divergence of the callitrichine lineage (14.8-16 MYA) and the AtelidaePitheciidae divergence (23-24.3 MYA). The molecular clock inferences were repeated three times to check the consistency of the results (Rutschmann, 2005).

Finally, to trace the evolution of SEC1 activity through the ancestral nodes, its condition in extant species was treated as a binary character, 0 being the "inactive" state and 1 the "active" state, and their distribution was plotted against the terminal taxons of the parsimony tree (Coddington, 1988; Carpenter, 1989; de Queiroz, 2000). The ancestral states on internal nodes were inferred using the software Mesquite 2.01 (Maddison and Maddison, 2007) by the likelihood criterion (Schluter et al., 1997; Pagel, 1999), using the previously obtained branch lengths. The "Asymmetrical Markov k-state 2 parameter" model of character evolution was adopted, with rates of forward and backward transformations of 8.87 and 8.07 , respectively. The root state frequencies were set to equilibrium, so they are assumed to be consistent with the model's rates, and the threshold for decision among the two states at each tree node was set to 1.0. Relative proportional likelihoods for each character state are depicted as small pie diagrams on the tree nodes, accompanied by the numeric value for the most likely state.

\section{RESULTS AND DISCUSSION}

Partial sequences were obtained for all samples, with estimated gene size ranging from 795 bp in Callithrix, to 1041 bp in Pithecia (Table 1). This variation is due to indel events, some of them shared within lineages, such as the callitrichines. The sequences of Callicebus brunneus, Brachyteles arachnoides, and Saimiri sciureus spp (Apoil et al., 2000) were also excluded from phylogenetic analysis due to the short fragments obtained; however, the latter two were used in the analysis of protein structure.

The nucleotide composition of SEC1 is marked by a high frequency of pyrimidines (54.6\%). The average GC content was $63.8 \%$ and the GC:AT proportion was 1.76 . These values are similar to those described for the FUT1 gene (Borges and Harada, 2004) and suggest that SEC1 is located in a GC-rich isochore, similar to that proposed by Sharp et al. (1995) for humans. The analysis of nucleotide substitution patterns revealed a predominance of transitions over transversions, with an average ratio of 2.73. Pairwise comparisons showed a slight predominance of $\mathrm{C} \leftrightarrow \mathrm{T}$ transitions, 1.14 times higher than $\mathrm{A} \leftrightarrow \mathrm{G}$. 
Table 1. Monkey samples, their respective GenBank accession numbers, sample origins, and number of base pairs sequenced.

\begin{tabular}{|c|c|c|c|}
\hline Primate group/species & Accession number & Origin $^{1}$ & Base pairs sequenced \\
\hline \multicolumn{4}{|l|}{ Family Atelidae } \\
\hline Alouatta belzebul & DQ166183 & Unknown & 608 \\
\hline Ateles belzebuth & DQ166185 & CENP, Brazil & 979 \\
\hline Ateles paniscus & DQ166186 & Rio Trombetas, Pará, Brazil & 991 \\
\hline Brachyteles arachnoides & DQ166187 & CPRJ, Brazil & 490 \\
\hline Lagothrix lagotricha & DQ166194 & Unknown & 967 \\
\hline \multicolumn{4}{|l|}{ Family Pitheciidae } \\
\hline Callicebus brunneus & DQ166188 & Rio Jamari, Rondônia, Brazil & 990 \\
\hline Callicebus brunneus & DQ166189 & Rio Jamari, Rondônia, Brazil & 704 \\
\hline Chiropotes satanas & DQ166192 & Unknown & 986 \\
\hline Chiropotes albinasus & DQ166193 & Unknown & 975 \\
\hline Pithecia irrorata & DQ166196 & Rio Jamari, Rondônia, Brazil & 1018 \\
\hline Pithecia irrorata & DQ166197 & Rio Jamari, Rondônia, Brazil & 1005 \\
\hline \multicolumn{4}{|l|}{ Family Cebidae } \\
\hline Aotus azarae & DQ166184 & Rio Jamari, Rondônia, Brazil & 991 \\
\hline Cebus olivaceus & DQ166191 & Rio Grande do Sul, Brazil & 990 \\
\hline Saimiri boliviensis boliviensis & DQ166200 & Bolivia & 995 \\
\hline \multicolumn{4}{|l|}{ Subfamily Callitrichinae } \\
\hline Callithrix humeralifer & DQ166190 & Unknown & 745 \\
\hline Saguinus fuscicollis weddelli & DQ166198 & Rio Jamari, Rondônia, Brazil & 979 \\
\hline Saguinus mystax & DQ166199 & CPRJ, Brazil & 989 \\
\hline Leontopithecus chrysomelas & DQ166195 & Unknown & 968 \\
\hline
\end{tabular}

${ }^{1} \mathrm{CENP}=$ Centro Nacional de Primatas; CPRJ $=$ Centro de Primatologia do Rio de Janeiro.

Comparisons among the $\mathrm{dN} / \mathrm{dS}$ gene ratios were made for the following primate groups (Schneider et al., 2001): Cebidae (Callitrichinae), Pitheciidae, Atelidae, and Platyrrhini. In all cases, the $\mathrm{dN} / \mathrm{dS}$ ratio was higher than 1, ranging from 1.18 in Atelidae to 1.58 in Pitheciidae (Table 2), slightly higher than those obtained in humans and Old World monkeys. The dN/dS ratio was also compared in different regions of SEC1: N-terminal region, transmembrane domain and C-terminal region. We observed that the $\mathrm{dN} / \mathrm{dS}$ ratio in the C-terminal region, where all protein domains were located, was similar to that described for the whole coding region (Table 2). The Z-test indicated positive selection, which can be interpreted as evidence of weak selective pressure in key regions for protein function, as proposed by Breton et al. (1998) and Martinez-Duncker et al. (2003).

\begin{tabular}{|c|c|c|c|c|}
\hline \multirow[t]{2}{*}{ Group } & \multicolumn{4}{|c|}{$\mathrm{dN} / \mathrm{dS}$} \\
\hline & Whole region & Coding N-terminal region & Transmembrane domain & C-terminal region \\
\hline Primates & 1.13 & 0.46 & 3.90 & 1.11 \\
\hline HOM & 0.91 & 0.26 & 0 & 0.91 \\
\hline OWM & 1.14 & 0 & 0 & 1.00 \\
\hline NWM & 1.32 & 0 & 0.37 & 1.32 \\
\hline Atelidae & 1.18 & $\mathrm{n} / \mathrm{c}$ & 0 & 1.18 \\
\hline Cebidae & 1.22 & 0 & 3.46 & 1.20 \\
\hline Pitheciidae & 1.58 & $\mathrm{n} / \mathrm{c}$ & 0.73 & 1.58 \\
\hline
\end{tabular}

$\mathrm{HOM}=$ Apes and humans; OWM = Old World monkeys; NWM = New World monkeys. $\mathrm{n} / \mathrm{c}=$ not computed. 
The alignment of nucleotide sequences showed two deletions (nucleotides 278-301 and 411-621) in Callithrix humeralifer. These deletions were previously described in Callithrix jacchus by Apoil et al. (2000). Other callitrichine genera also present deletion events. Saguinus sequences show a single 2-bp deletion at positions 408-409, and in Leontopithecus there is a 1-bp deletion at position 566 and a 4-bp deletion at position 1095-1098. Insertion events were observed in Pithecia irrorata at position 121-141 (21 bp) and 354-357 (4 bp); in Callithrix humeralifer at 1022-1023 (2 bp), 1036-1041 (6 bp) and 699. The latter also occurs in Aotus azarae.

The predicted protein of these species (Table 3 ) lacks similarity with other fucosyltransferases from residues 90 in Callithrix jacchus, 93 in Callithrix humeralifer, 119 in Pithecia irrorata, 135 in Saguinus, 188 in Leontopithecus and 232 in Aotus azarae. Consequently, they lack the three conserved domains proposed by Oriol et al. (1999) for all $\alpha 1,2$ FUT, except for that of Callithrix. Histidine and arginine residues (positions 188 and 191 in Neotropical primates), related by Takahashi et al. (2000) as important for $\alpha 1,2$ and $\alpha 1,6$ FUT enzymatic activity, are also not conserved in these species. In addition, these species have multiple stop codons, except for Callithrix humeralifer. These results strongly suggest that the predicted protein has no activity. In the remaining primate sequences, the domains are relatively conserved, suggesting that, despite $\mathrm{dN} / \mathrm{dS}$ results indicating positive selection, SEC1 in all other New World monkeys produces a fully active protein.

Our results indicate that the proposed inactivation of SEC1 in callitrichines, Aotus and Pithecia, as well as in apes (Homo, Pan and Gorilla), may have evolved by independent transformations. Also, Pithecia irrorata, Saguinus and Aotus show a high similarity in the carboxy portion of the human and gorilla protein, including a stop codon at position 276 of the alignment.

The $\alpha 1,2$ FUT are widely expressed in vertebrates, invertebrates, plants, and bacteria. They are involved in the final stages of the synthesis of human $\mathrm{H}$ histo-blood group antigens associated with the $\mathrm{ABO}$ system and in a variety of pathological processes as many parasites, bacteria and viruses are know to bind to carbohydrate structures in their effort to initiate the infection process (Costache et al., 1997; Ma et al., 2006).

During the evolutionary process, the $\alpha 1,2$ FUT showed a progress from endodermal to ectodermal tissues. In humans, the erythrocytes (mesodermal origin) were the last cells to acquire the histo-blood group ABH antigens (Barreaud et al., 2000).

In Catarrhini, only two $\alpha 1,2$ FUT (FUT1 and FUT2) are expressed, with the SEC1 gene inactive due to an accumulation of mutations, like deletions and stop codons (Apoil et al., 2000). However, this non-functional gene does not alter the expression of the $\mathrm{H}$ antigens, because the other two are responsible for protein expression in secretions and cell surfaces (Mollicone et al., 1995; Costache et al., 1997).

The Platyrrhini species possess the three $\alpha 1,2$ FUT genes, and it is known that their expression is quite different from that observed in the Catarrhini, the $\mathrm{H}$ antigen being present only in secretions; it is replaced in red blood cells by the $\alpha$-Gal antigen (Oriol et al., 1992; Apoil et al., 2000). Nevertheless, no mutation was found in the FUT1 gene that could explain the lack of expression (Borges and Harada, 2004). Apoil et al. (2000) suggest that an Alu-like element inserted in a region crucial for regulation is responsible for expression of the $\mathrm{H}$ enzyme in human and ape erythroid lineages. The same was proposed for bovines and humans, in which SEC1 gene expression was detected only in intestinal tract cells (Barreaud et al., 2000). 


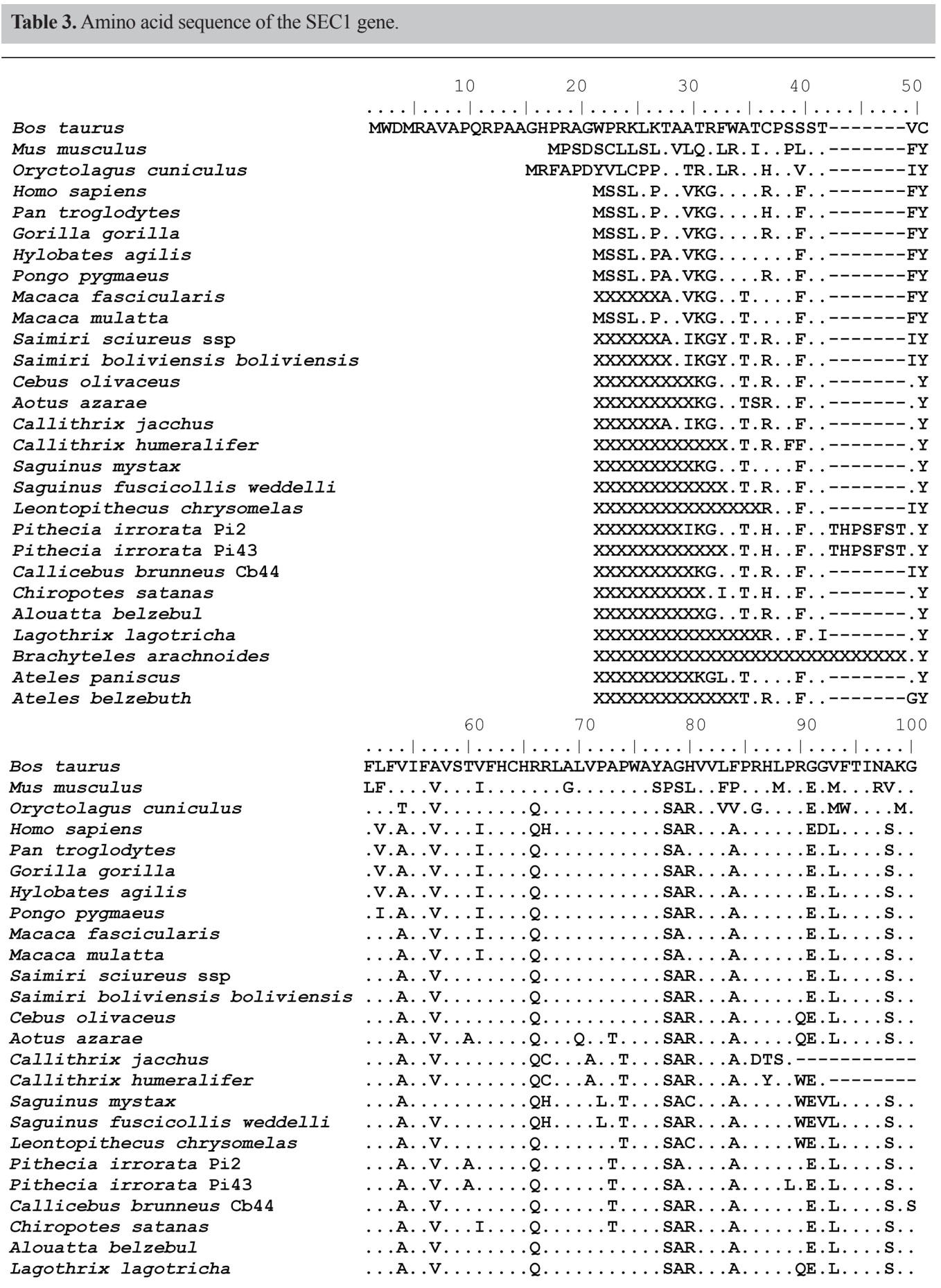

Continued on next page 
Table 3. Continued.

Brachyteles arachnoides
Ateles paniscus
Ateles belzebuth

Bos taurus
Mus musculus
Oryctolagus cuniculus
Homo sapiens
Pan troglodytes
Gorilla gorilla
Hylobates agilis
Pongo pygmaeus
Macaca fascicularis
Macaca mulatta
Saimiri sciureus ssp
Saimiri boliviensis boliviensis
Cebus olivaceus
Aotus azarae
Callithrix jacchus
Callithrix humeralifer
Saguinus mystax
Saguinus fuscicollis weddelli
Leontopithecus chrysomelas
Pithecia irrorata Pi2
Pithecia irrorata Pi43
Callicebus brunneus Cb44
Chiropotes satanas
Alouatta belzebul
Lagothrix lagotricha
Brachyteles arachnoides
Ateles paniscus
Ateles belzebuth

Bos taurus

Mus musculus

Oryctolagus cuniculus

Homo sapiens

Pan troglodytes

Gorilla gorilla

Hylobates agilis

Pongo pygmaeus

Macaca fascicularis

Macaca mulatta

Saimiri sciureus ssp

Saimiri boliviensis boliviensis

Cebus olivaceus

Aotus azarae

Callithrix jacchus

Callithrix humeralifer

Saguinus mystax

Saguinus fuscicollis weddelli

Leontopithecus chrysomelas

Pithecia irrorata Pi2

Pithecia irrorata $\mathrm{Pi} 43$

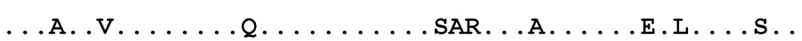

$\ldots$. A. . . . . . . . . . . . . . SAR . . . . . E. L. . . . .

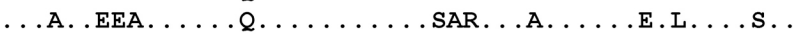

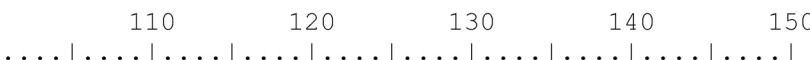

RLGNQMGEYATLYALAKMNGRAAFIPPQMHSTLAPIFRITLPVLHDATAR

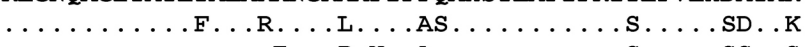
$\ldots \ldots \ldots \ldots$. . . . . . . . . . . . . . S

$\ldots \ldots \ldots \ldots \ldots$. . . A . RV . . . . . . . . .

$\ldots \ldots \ldots \ldots \ldots \ldots \ldots \ldots \ldots \ldots \ldots \ldots$. . . . . .

$\ldots \ldots \ldots \ldots \ldots \ldots \ldots$. . . . . . . . . .

$\ldots \ldots \ldots \ldots \ldots \ldots \ldots \ldots \ldots \ldots \ldots \ldots \ldots$. . . .

$\ldots \ldots \ldots \ldots \ldots \ldots \ldots \ldots \ldots \ldots \ldots \ldots \ldots \ldots \ldots$.

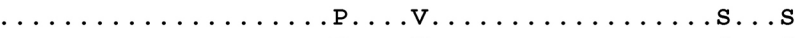

$\ldots \ldots \ldots \ldots \ldots \ldots \ldots \ldots \ldots$. . . . . . .

$\ldots \ldots \ldots \ldots$. . . . . . . . . . . . . . . SS

$\ldots \ldots \ldots$. . . . . . A. . . . . . . . . . . .

$\ldots \ldots \ldots \ldots$. . . . . . . . . . . . . . ST.S

$\ldots \ldots$. . . . . . . . . A . . V. . V. . . . . . ST . S . . WG- W --------------------RLG----------------NQMGEY . T

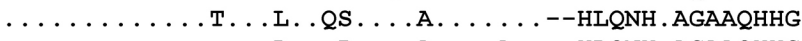
$\ldots \ldots \ldots \ldots \ldots$. . . . . . . . . . --HLQNH. AGAAQHHG H. ........ . . . . . ... . . . . . . . . . . . . S . . . . . . . . . . LGE . . CLH. SP. AQRPLPHLQNH. AGAAQHHS $\ldots \ldots \ldots \ldots$. . . . . . CLE . SP . AQRPLPHLQNH. AGAAQHHS $\ldots \ldots \ldots \ldots$. . . . . . . . . . . . . . . . . . . . S $\ldots \ldots \ldots$. . . . . . . . . . . . . . . . . $\ldots \ldots \ldots$.................... . L. .................. . . . . . . . . S $\ldots \ldots \ldots \ldots$. . . . . . . . . . . . . . . $\ldots \ldots \ldots$. . . . . . . . . . . . . . . . $\ldots \ldots \ldots$......................ST $\begin{array}{lllll}160 & 170 & 180 & 190 & 200\end{array}$ $\ldots|\ldots| \ldots|\ldots| \ldots|\ldots| \ldots|\ldots| \ldots|\ldots| \ldots|\ldots|$ SVPWQNYHLNDWMEEQYRH IPGEYVRLTGYPCSWTFYHHLRAE ILQEFTL RI . . . . . . . . . . . . . . . . . . . . . . . . .

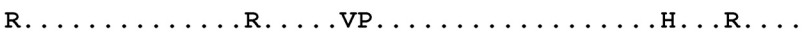
RI. . . . . . . . . RC.H. . . . . . . . . . .

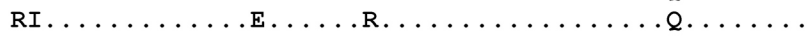
RI . . . . . E. . . R. . . . . . . . . . . . RI. . . . . . E.H. . R. . . . . . . . . CH. . . . RI . . . . . EE. . . R. . . . . . . . . . . RI ................R. . . . . . . . . . . . . .

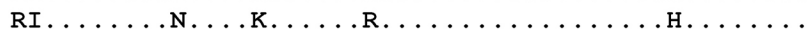
RI.........E.H. . R. . . . . . . . . . . . . . . RI. . . . . . . . . . R. . . . . . . . . . . . . . . . . RI ........E. . . R. . . . . . . . . . . . . . RI......... K. . . . H. C. . . . . . . . . . . . CM. . P---------------S*MG I L. S. RPRCTAP . ------LYALA-----------------KLNGRPAFIPAQI . STT--------QQDPLAELPPERIDGRGIPPHPGALCPPHGLPQLLDLIPPPPPGDPPGVH QQDPLVELPPERLDGRGVPPHPRALCPPHGLPQLLDLLPPPPPGDPPGVH RI . . . . .....E.H..I.R. .H. .... . . . TTSARRSSRSSPC QQDPLAELPPERLDGRGVPPHPGALCSPHGLPQLLDLLPPPPPRDPPGVH QQDPLAELPPERLDGRGVPPHPGALCSPHGLPQLLDLLPPPSPRDSPGVH

Continued on next page

Genetics and Molecular Research 7 (3): 663-678 (2008)

CFUNPEC-RP www.funpecrp.com.br 
Table 3. Continued.

Callicebus brunneus Cb44
Chiropotes satanas
Alouatta belzebul
Lagothrix lagotricha
Brachyteles arachnoides
Ateles paniscus
Ateles belzebuth

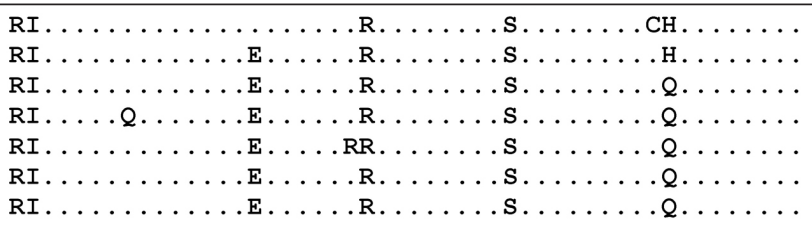

DII

$260 \quad 270 \quad 280 \quad 290 \quad 300$
$\ldots|\ldots| \ldots|\ldots| \ldots|\ldots| \ldots|\ldots| \ldots|\ldots| \ldots|\ldots|$ GYLQQALDWFRARHHS PLFVITSDDMAWCRRNINSSHRDVVFAGSGQQGS

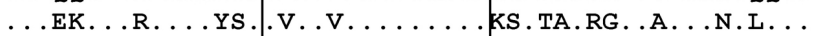
... . . . . . PTAP.V. .V. .NG . . . E . DA.RG . . . . . LE . -- . PA. GPGLVPGLLP. PGLCGHQR * HGLVPGEHQQLPWGRGVRWQWPPG

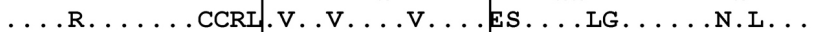
QG. PA. GPGLVPGLLP. PGLCGHQR * HGLVPGEHQQLPWGRGVRWQWPPG

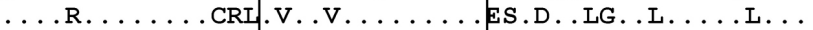
$\ldots$. R. . . . . . CRI . V. . V. . . . . . . . . . L . . . . N.L. .

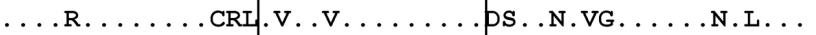

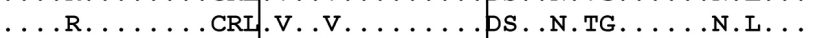
????????????????]?????????????????????????????????

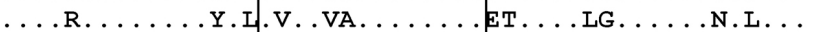
$\ldots$. R. . . . . YRL.V. .VA. . . . . ET . . . LG . . . . N.L. . PG. PA. GPGLVPGPLI. PGLCGRQQ*HGLVPGNHQQLPWGRGICREWPPG

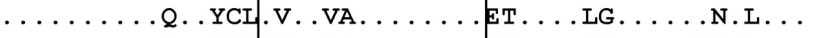
$\ldots \ldots$. . . . YCL.V. VA. . . . E . . . LW. . . RN.L. .

Continued on next page 
Table 3. Continued.

Saguinus mystax
Saguinus fuscicollis weddelli
Leontopithecus chrysomelas
Pithecia irrorata $\mathrm{Pi} 2$
Pithecia irrorata $\mathrm{Pi} 43$
Callicebus brunneus $\mathrm{Cb} 44$
Chiropotes satanas
Alouatta belzebul
Lagothrix lagotricha
Brachyteles arachnoides
Ateles paniscus
Ateles belzebuth

Bos taurus

Mus musculus

Oryctolagus cuniculus

Homo sapiens

Pan troglodytes

Gorilla gorilla

Hylobates agilis

Pongo pygmaeus

Macaca fascicularis

Macaca mulatta

Saimiri sciureus ssp

Saimiri boliviensis boliviensis

Cebus olivaceus

Aotus azarae

Callithrix jacchus

Callithrix humeralifer

Saguinus mystax

Saguinus fuscicollis weddelli

Leontopithecus chrysomelas

Pithecia irrorata $\mathrm{Pi} 2$

Pithecia irrorata Pi43

Callicebus brunneus $\mathrm{Cb} 44$

Chiropotes satanas

Alouatta belzebul

Lagothrix lagotricha

Brachyteles arachnoides

Ateles paniscus

Ateles belzebuth
PG. PA. GPGLVPGPLP|. PSLCGRQR * HGLVTPGNRQQLPWGRGFCREWPPG PG. PA. GPGLVPGPLP. PSLCGRQR * HGLVPGNHQQLPWGRGFCREWPPG ATCSGPWTGSGPVTT . WSLWSPAMTWPGAGKPSTAP SGMWFLP . MASRAH PG . PA. GPGLVPGPLP. PSLCGHQR*RGLVPGNYQQLPRGCGVRREWPPG PG. PA. GPGLVPGPLP. PSLCGHQR * HGLVPGNYQQLPRGCGVRREWPPG ...R. . . . . . YRI.V. .VA. . . . . ET . . . LG. . . . . . LK. . . . . R. . . . . . YRL.V. .VA. . . . . . ET . . . LG . . . . D.L. . ????????????????]????????????????????????????????? . . . R. . . . . YRL.V. .VA. . . . . ET . . . LG . . . . N.LK. . ????????????????]?????????????]???????????????????

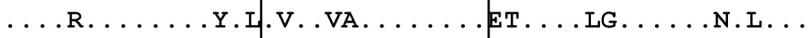
...R. . . . . . YRL.V. .VA. . . . . . ET. . . LG. . . . . . . .

\section{DIII}

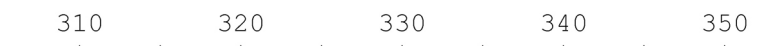

$\ldots \ldots|\ldots| \ldots|\ldots| \ldots|\ldots| \ldots|\ldots| \ldots|\ldots| \ldots|\ldots|$ PARDFALITQCNHTVITVGTFGIWAAYLAGGSTVYLANFTLPGSRFRMIF ..K.I...M. . . ... . . . . . T. . . . . . Q.N.P.HTV. . K. . . . . . . I. I. . . . . T. . . . . Y.A.D.P.HLV. LTCQGLRTAHTVQPHHHHRGHLRGLGRVPR . RGHC . PGQLH . AQLPFQRG ..K. . . . . . I. . . . V. . . . . . . . . . . . P.NVV. LTCQGLRTAHTVQPHHHHCGHLRGLGRVPR. RGHCQPGQLH . AQLPFQRG

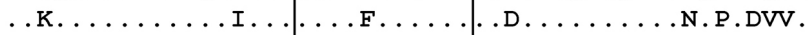
.K. . . . . I....V. . . . . D. . . . . N.P.DVV.

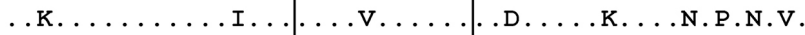
KK. . . . . . I I. . . . . . . . . . . . . . N.P.N.V. ?????????????????????????????????????????????????? . . . . . . . . . . . . . . . . D. I . . . R.H.P.NLV. $\ldots \ldots \ldots$. . . . . . . . . . . T. . . . . . R. TPPSTWSL LTCQGLCTADTGQPHHHHCGHLWNLGC. PH . WGHH . PGQLHTAALPFRPG

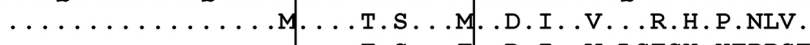
$\ldots \ldots \ldots \ldots \ldots \ldots \ldots$. . . . . . . . . I. .V.LSTSH.HTPPST LTCQGLCTADTVQPHCHHRGHLWNLGC . PH . RGHH . PGQLHTAALPLQPG LTCQGLCTADTVQPHCHHRGHLWNLGC . PH . RGHH . PGQLHTAALPLQPG LPGTLHC *HSATTPSSLWAPLEPGPPTSRA. TPSTWPTSHSHTPPSTWSL LTCQGLCTSDTVQPHHHHRGHLWNLGR . PHRWGHH . PGQLH . APLPLRPG LTCQGLCTSDTVQPHHHHRGHLWNLGR . PHRWGHH . PGQLH . APLPLRPG

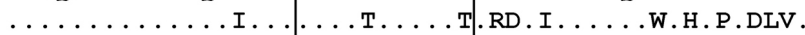

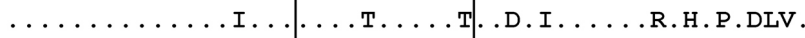
??????????????????????????????????????????????????

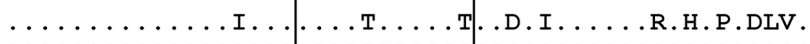
?????????????????????????????????????????????????? . . . . . . . . . . . . . . . . . . I. . . R.H.P.DLV. $\ldots \ldots$. . . . . . . . . . . . I. . . R.R.P.CLV.

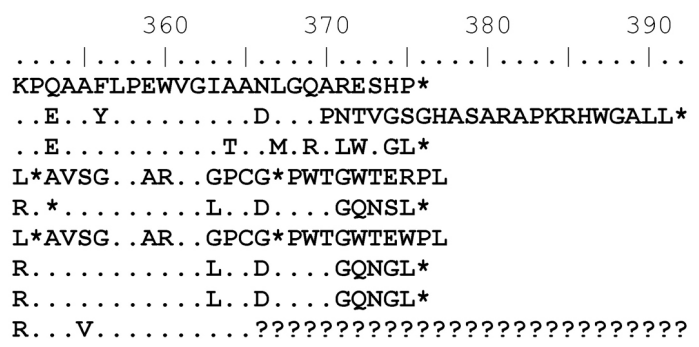

Continued on next page 
Table 3. Continued.

\begin{tabular}{|c|c|}
\hline Macaca mulatta & R. . . . . . . . . . . . . GQNGL* \\
\hline Saimiri sciureus ssp & \\
\hline Saimiri boliviensis boliviensis & R........M. .??????????????????????????? \\
\hline Cebus olivaceus & GRKQPSCQSGWAWRL?? ?? ???? ????? ?????????????? \\
\hline Aotus azarae & L*ATSSF .AT.RGHGG* \\
\hline Callithrix jacchus & R.......M. .??????????????????????????? \\
\hline Callithrix humeralifer & LSLGHKQ . SCQSGW??? ?? ???? ???? ??????????????? \\
\hline Saguinus mystax & L*ATSS. . AR. . GHGG* \\
\hline Saguinus fuscicollis weddelli & L*ATSS . AR . . GHGG* \\
\hline Leontopithecus chrysomelas & GHKQLSCQSGH. G* \\
\hline Pithecia irrorata $\mathrm{Pi} 2$ & LWTTSS. . AR. . GHGG* \\
\hline Pithecia irrorata $\mathrm{Pi} 43$ & LWTTSS. . AR . . GHGG* \\
\hline Callicebus brunneus $\mathrm{Cb} 44$ & R.......M. .??????????????????????????? \\
\hline Chiropotes satanas & R.......M. .??????????????????????????? \\
\hline Alouatta belzebul & \\
\hline Lagothrix lagotricha & R.......M. (M.???????????????????????????? \\
\hline Brachyteles arachnoides & 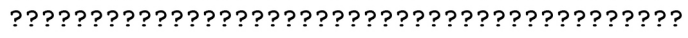 \\
\hline Ateles paniscus & R......M.M. .??????????????????????????? \\
\hline Ateles belzebuth & R........M. .??????????????????????????? \\
\hline
\end{tabular}

Dots represent identity, dashes represent amino acid deletions, " $X$ " represents unknown residues, asterisks $(*)$ indicate stop codons, and question marks (?) indicate missing data. Boxes indicate the three domains present in all $\alpha 1,2$ fucosyltransferases. Residue numbers are indicated above the reference sequences for the alignment. DI $=$ conserved domain I, DII = conserved domain II; DIII = conserved domain III.

Comparison of SEC1 gene evolutionary rates among Atelidae, Cebidae and Pitheciidae families showed variation ranging from $0.791 \times 10^{-9}$ (Atelidae) to $1.314 \times 10^{-9}$ (Cebidae) (Table 4), while in all New World monkeys the overall ratio was $1.074 \times 10^{-9}$. This difference could be explained by several changes in the nucleotide sequences (indels), especially in callitrichines, which may increase the rate in the cebids compared to other groups. This is supported by the results of the relative rate test, which indicated significant differences in the evolutionary rates $(\mathrm{P}<0.05)$ in Aotus, callitrichines and Pithecia when compared to other primates.

\begin{tabular}{lccc}
\multicolumn{2}{l}{ Table 4. Estimates of the rates of evolution $(\mathrm{r}=\mathrm{K} / 2 \mathrm{~T})$ of the SEC1 gene in the different groups of primates. } \\
\hline Group & $\mathrm{K}$ & $\mathrm{T}$ & Substitutions/site/year $(\mathrm{r})$ \\
\hline Primates & 0.0659 & 63 & $0.523 \times 10^{-9}$ \\
HOM & 0.0332 & 18 & $0.922 \times 10^{-9}$ \\
OWM & 0.0247 & 14 & $0.881 \times 10^{-9}$ \\
NWM & 0.0537 & 26 & $1.033 \times 10^{-9}$ \\
Atelidae & 0.0253 & 15.8 & $0.800 \times 10^{-9}$ \\
Cebidae & 0.0578 & 20.2 & $1.431 \times 10^{-9}$ \\
Callitrichinae & 0.0625 & 14.2 & $2.200 \times 10^{-9}$ \\
Pitheciidae & 0.0404 & 21.3 & $0.948 \times 10^{-9}$ \\
\hline
\end{tabular}

Divergence times (T) are given in millions of years ago, according to Opazo et al. (2006), for New World monkeys and Goodman et al. (1998) for other primates. HOM = Apes and humans; OWM = Old World monkeys; NWM = New World monkeys.

Except in Cebidae, in which evolutionary rates described here are closer to those reported for $\psi \beta$-globin $\left(2.0 \times 10^{-9}\right.$, in primates) and $\psi \eta$-globin $\left(1.9 \times 10^{-9}\right.$ in Aotus $)$ (Li et al., 1981; Li and Gojobori, 1983; Goodman et al., 1984; Harris et al., 1984), all other values were closer to that reported for FUT1 $\left(0.739 \times 10^{-9}\right)$ in these primate groups, which is relatively 
lower than the evolutionary rates of pseudogenes (Borges and Harada, 2004).

The SEC1-like family presents higher evolutionary rates than the FUT2-like family due to accumulation of mutations, such as premature stop codons, in the SEC1 pseudogene (Apoil et al., 2000). These mutations are also observed in some Old and New World monkeys, but until now no SEC1 transcript has been found in these species or in healthy human tissues. Besides the mutations described, probably a DNA rearrangement at the SEC1 locus occurred during and/or after a retrotransposition event in humans. It is widely known that subsequent mutations are facilitated by the previous gene-inactivating rearrangement (Saunier et al., 2001).

The phylogenetic tree obtained for the SEC1 gene (Figure 1) is basically consistent with published arrangements, except for Callicebus, which grouped within the Atelidae (bootstrap support $=76 \%$ ), instead of within the Pitheciidae (e.g., Harada et al., 1995; Porter et al., 1997; Goodman et al., 1998; Schneider, 2000; Schneider et al., 1993, 1996, 2001; Schrago, 2007).

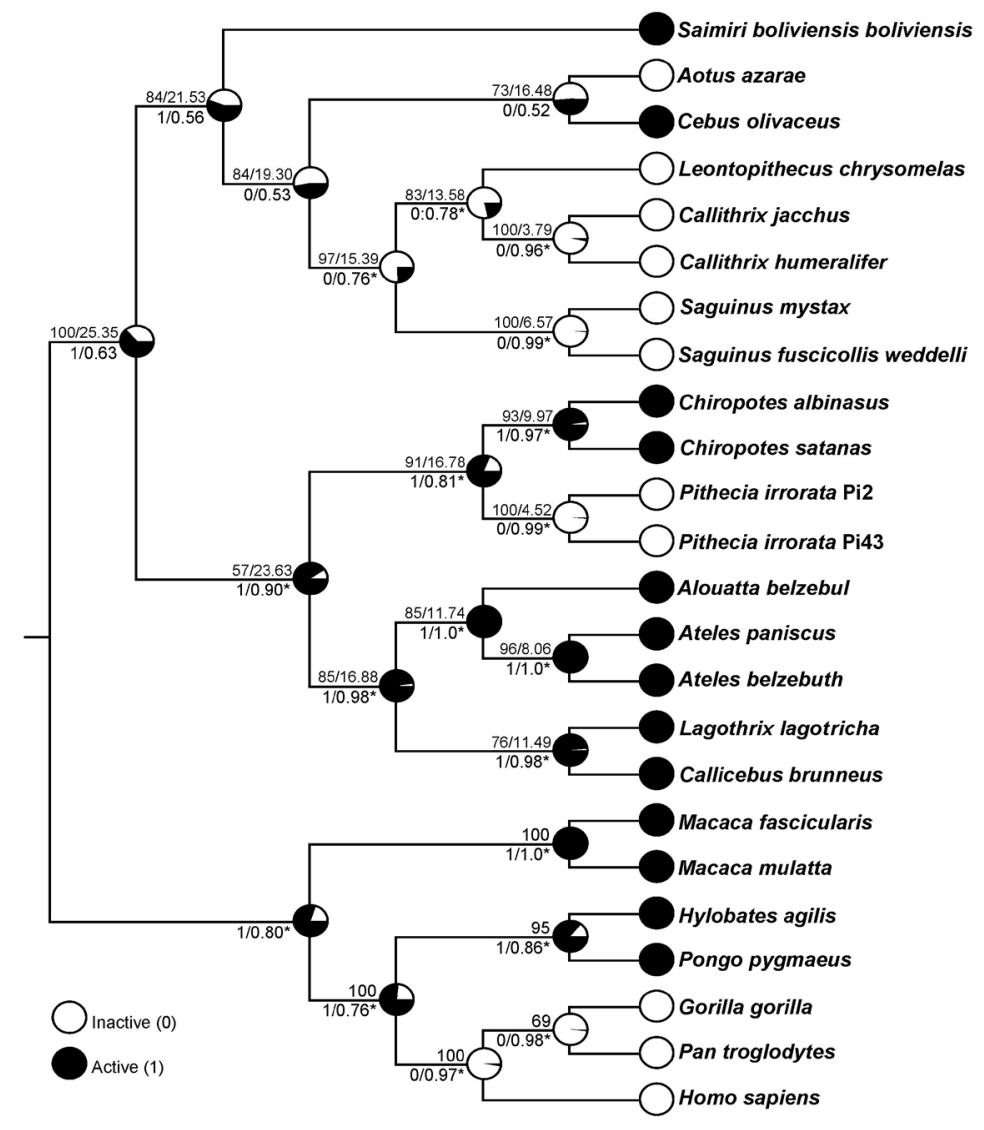

Figure 1. Phylogenetic tree of the SEC1 gene inferred through the maximum parsimony method. The pie diagrams at the nodes denote the proportional likelihoods for each state of the binary character "SEC1 gene activity". The numbers associated with the internal branches correspond to character state/character state likelihood. Bootstrap values/divergence times are above the branches. Asterisks denote unambiguous choice of ancestral states, according to the decision threshold. Ancestral states inference $-\log L=13.864273495$. 
The transition/transversion rates and nucleotide sequence alignment support the hypothesis that primate SEC1 evolved by divergent evolution, as proposed for the origin of fucosyltransferases (Breton et al., 1998; Oriol et al., 1999; Barreaud et al., 2000; Bureau et al., 2001; Borges and Harada, 2004). In contrast, multiple stop codons and amino acid changes present in different species suggest convergent inactivation events in apes, Callitrichinae, Aotus and Pithecia.

Considering the stop codon detected in SEC1 gene of callitrichines, Aotus and Pithecia, an enzyme that lacks at least 131 amino acids is produced in comparison with the functional ones, suggesting the non-functionality. This hypothesis is supported by the description of reduced activity of different fucosyltransferases induced by deletion of one or more amino acids in the carboxy-terminal region (Xu et al., 1996).

Based on Bayesian analysis of divergence times (Table 5), the differentiation of the Platyrrhini SEC1 lineage occurred at approximately 25.35 MYA. This is consistent with reported values for New World monkey radiation, which range from 20 to 35 MYA (Goodman et al., 1998; Schneider, 2000; Schneider et al., 1993, 2001; Figueiredo, 2006; Schrago, 2007). Likelihood inference of ancestral states for the activity of SEC1 gene in New World monkeys (Figure 1) indicated that the inactive condition evolved independently at least twice in the Platyrrhini.

\begin{tabular}{|c|c|c|c|c|c|c|}
\hline Lineage & Present study & $\begin{array}{c}\text { Goodman } \\
\text { et al. (1998) }\end{array}$ & $\begin{array}{l}\text { Schneider } \\
\text { et al. (2001) }\end{array}$ & $\begin{array}{l}\text { Figueiredo } \\
\text { (2006) }\end{array}$ & $\begin{array}{c}\text { Opazo } \\
\text { et al. }(2006)\end{array}$ & $\begin{array}{l}\text { Schrago } \\
(2007)\end{array}$ \\
\hline Platyrrhini & $25.35(23.89-28.8)$ & 25 & 26 & $30.4(24.2-42.2)$ & 26 & $20.1(15.6-28.3)$ \\
\hline Atelidae & $16.88(11.27-22.49)$ & 16 & 15 & $21.1(17.1-29.5)$ & 16.75 & $12.4(9.1-18.6)$ \\
\hline Cebidae & $21.53(20.28-22.73)$ & 22 & 23 & $26.4(20.7-36.6)$ & 22.75 & $16.9(12.7-23.3)$ \\
\hline Callitrichinae & $15.39(14.83-15.96)$ & 13 & 16 & $18.0(13.8-25.4)$ & 16 & $11.8(8.3-17.4)$ \\
\hline Chiropotes-Pithecia & $16.78(10.99-22.25)$ & 10 & 11 & $15.6(11.7-22.0)$ & 11.96 & $9.1(6.4-13.4)$ \\
\hline
\end{tabular}

The inactive condition of SEC1 proposed for Pithecia irrorata could not be traced back to the last common ancestor with Chiropotes. Consequently, it probably evolved in the Pithecia lineage, at about 4.62 MYA. In the Aotus-Cebus-Callitrichinae group, it is not evident whether SEC1 inactivation occurred independently in Aotus and Callitrichinae lineages or in the last common ancestor of the group. In this latter case, the active condition in Cebus would be a reversion (Figure 1). Despite the inactive state observed in the last common ancestor of this group, the nucleotide changes leading to this condition seem to be independent with different indel events responsible for gene inactivation in Aotus, Leontopithecus, Saguinus (with considerable changes in the open reading frame) and in Callithrix.

The similarity of the carboxy-portion of the amino acid sequences, especially at domains II and III (Table 3) amongst Callithrix and hypothetically active in the New World monkeys, suggests that a great deletion event occurred at domain II, followed by other events (including a premature stop codon mutation in Callithrix jacchus), which would be responsible for the inactivation of the protein. In our tree, one of the main differences in relation to other phylogenies is the arrangement of the Cebidae. In other molecular phylogenies of primates, the position of Aotus, Cebus and Saimiri species varies in relation to other Callitrichinae (e.g., Harada et al., 1995; Porter et al., 1997; Goodman et al., 1998; Schneider, 2000; Schneider et al., 1996, 2001; Opazo et al., 2006; Schrago, 2007). This could be a result of the rapid divergence events that occurred in the Cebidae, which would decrease the resolution 
within this group. Although in our tree, Aotus grouped with Cebus, both Cebus and Saimiri share an active state for SEC1.

In conclusion, our proposition is the inactivity of SEC1 in some New World monkey lineages, a feature that could have evolved independently more than once. Further analyses of protein expression and tertiary structure, as well as new DNA sequences that include promoter regions will help to clarify this subject.

\section{ACKNOWLEDGMENTS}

We are thankful to Mr. Arlindo Pinto de Souza Junior for technical support. Research supported by grants from the Universidade Federal do Pará (UFPA).

\section{REFERENCES}

Apoil PA, Roubinet F, Despiau S, Mollicone R, et al. (2000). Evolution of alpha 2-fucosyltransferase genes in primates: relation between an intronic Alu-Y element and red cell expression of ABH antigens. Mol. Biol. Evol. 17: 337-351.

Barreaud JP, Saunier K, Souchaire J, Delourme D, et al. (2000). Three bovine alpha2-fucosyltransferase genes encode enzymes that preferentially transfer fucose on Galbeta1-3GalNAc acceptor substrates. Glycobiology 10: 611-621.

Borges BN and Harada ML (2004). Divergent evolution and purifying selection of the H (FUT1) gene in New World monkeys (Primates, Platyrrhini). Genet. Mol. Biol. 27: 342-349.

Breton C, Oriol R and Imberty A (1998). Conserved structural features in eukaryotic and prokaryotic fucosyltransferases. Glycobiology 8: 87-94.

Bureau V, Marionneau S, Cailleau-Thomas A, Le Moullac-Vaidye B, et al. (2001). Comparison of the three rat GDP-Lfucose:beta-D-galactoside 2-alpha-L-fucosyltransferases FTA, FTB and FTC. Eur. J. Biochem. 268: 1006-1019.

Carpenter JM (1989). Testing scenarios: Wasp social behavior. Cladistics 5: 131-144.

Coddington JA (1988). Cladistic tests of adaptational hypotheses. Cladistics 4: 3-22.

Costache M, Cailleau A, Fernandez-Mateos P, Oriol R, et al. (1997). Advances in molecular genetics of alpha-2- and alpha-3/4-fucosyltransferases. Transfus. Clin. Biol. 4: 367-382.

de Queiroz K (2000). Logical problems associated with including and excluding characters during tree reconstruction and their implications for the study of morphological character evolution. In: Phylogenetic Analysis of Morphological Data (Wiens JJ, ed.). Smithsonian Institution Press, Washington, DC, 192-212.

Farris JS (1969). A successive approximations approach to character weighting. Syst. Zool. 18: 374-385.

Felsenstein J (1985). Confidence limits on phylogenies: An approach using the bootstrap. Evolution 39: 783-791.

Figueiredo WMB (2006). Estimativas de Tempo de Divergência em Platirrinos e Filogenia Molecular e Filogeografia dos Uacaris, Parauacus e Cuxius. Doctoral thesis, Universidade Federal do Pará, Conselho Nacional de Desenvolvimento Científico e Tecnológico, Pará.

Goodman M, Koop BF, Czelusniak J and Weiss ML (1984). The eta-globin gene. Its long evolutionary history in the betaglobin gene family of mammals. J. Mol. Biol. 180: 803-823.

Goodman M, Porter CA, Czelusniak J, Page SL, et al. (1998). Toward a phylogenetic classification of primates based on DNA evidence complemented by fossil evidence. Mol. Phylogenet. Evol. 9: 585-598.

Hall TA (1999). BioEdit: a user-friendly biological sequence alignment editor and analysis program for Windows 95/98/ NT. Nucleic Acids Symp. Ser. 41: 95-98.

Harada ML, Schneider H, Schneider MP, Sampaio I, et al. (1995). DNA evidence on the phylogenetic systematics of New World monkeys: support for the sister-grouping of Cebus and Saimiri from two unlinked nuclear genes. Mol. Phylogenet. Evol. 4: 331-349.

Harris S, Barrie PA, Weiss ML and Jeffreys AJ (1984). The primate psi beta 1 gene. An ancient beta-globin pseudogene. J. Mol. Biol. 180: 785-801.

Hasegawa M, Kishino H and Yano T (1985). Dating of the human-ape splitting by a molecular clock of mitochondrial DNA. J. Mol. Evol. 22: 160-174.

Hitoshi S, Kusunoki S, Kanazawa I and Tsuji S (1995). Molecular cloning and expression of two types of rabbit betagalactoside alpha 1,2-fucosyltransferase. J. Biol. Chem. 270: 8844-8850. 
Hitoshi S, Kusunoki S, Kanazawa I and Tsuji S (1996). Molecular cloning and expression of a third type of rabbit GDPL-fucose:beta-D-galactoside 2-alpha-L-fucosyltransferase. J. Biol. Chem. 271: 16975-16981.

Javaud C, Dupuy F, Maftah A, Julien R, et al. (2003). The fucosyltransferase gene family: an amazing summary of the underlying mechanisms of gene evolution. Genetica 118: 157-170.

Kelly RJ, Rouquier S, Giorgi D, Lennon GG, et al. (1995). Sequence and expression of a candidate for the human secretor blood group alpha(1,2)fucosyltransferase gene (FUT2). Homozygosity for an enzyme-inactivating nonsense mutation commonly correlates with the non-secretor phenotype. J. Biol. Chem. 270: 4640-4649.

Kimura M (1980). A simple method for estimating evolutionary rates of base substitutions through comparative studies of nucleotide sequences. J. Mol. Evol. 16: 111-120.

Kishino H, Thorne JL and Bruno WJ (2001). Performance of a divergence time estimation method under a probabilistic model of rate evolution. Mol. Biol. Evol. 18: 352-361.

Kitching IJ, Forey PL, Humphries CJ and Williams DM (1998). Cladistics: the theory and practice of parsimony analysis. Oxford University Press, Oxford.

Kumar S, Tamura K and Nei M (2004). MEGA3: Integrated software for Molecular Evolutionary Genetics Analysis and Sequence Alignment. Brief. Bioinform. 5: 150-163.

Li WH (1997). Molecular Evolution. Sinauer Associates, Sunderland.

Li WH and Gojobori T (1983). Rapid evolution of goat and sheep globin genes following gene duplication. Mol. Biol. Evol. 1: 94-108.

Li WH, Gojobori T and Nei M (1981). Pseudogenes as a paradigm of neutral evolution. Nature 292: 237-239.

Ma B, Simala-Grant JL and Taylor DE (2006). Fucosylation in prokaryotes and eukaryotes. Glycobiology 16: 158R-184R.

Maddison WP and Maddison DR (2007). Mesquite: a modular system for evolutionary analysis. http://mesquiteproject.org.

Martinez-Duncker I, Mollicone R, Candelier JJ, Breton C, et al. (2003). A new superfamily of protein-O-fucosyltransferases, alpha2-fucosyltransferases, and alpha6-fucosyltransferases: phylogeny and identification of conserved peptide motifs. Glycobiology 13: 1C-5C.

Mollicone R, Cailleau A and Oriol R (1995). Molecular genetics of H, Se, Lewis and other fucosyltransferase genes. Transfus. Clin. Biol. 2: 235-242.

Nei M and Kumar S (2000). Molecular Evolution and Phylogenetics. Oxford University Press, New York.

Nixon KC (1999). The Parsimony Ratchet, a new method for rapid parsimony analysis. Cladistics 15: 407-414.

Nixon KC and Carpenter JM (1993). On outgroups. Cladistics 9: 413-426.

Opazo JC, Wildman DE, Prychitko T, Johnson RM, et al. (2006). Phylogenetic relationships and divergence times among New World monkeys (Platyrrhini, Primates). Mol. Phylogenet. Evol. 40: 274-280.

Oriol R, Mollicone R, Coullin P, Dalix AM, et al. (1992). Genetic regulation of the expression of ABH and Lewis antigens in tissues. APMIS 100 (Suppl. 27): 28-38.

Oriol R, Mollicone R, Cailleau A, Balanzino L, et al. (1999). Divergent evolution of fucosyltransferase genes from vertebrates, invertebrates, and bacteria. Glycobiology 9: 323-334.

Pagel M (1999). The maximum likelihood approach to reconstructing ancestral character states of discrete characters on phylogenies. Syst. Biol. 48: 612-622.

Paulson JC and Colley KJ (1989). Glycosyltransferases. Structure, localization, and control of cell type-specific glycosylation. J. Biol. Chem. 264: 17615-17618.

Pond SL, Frost SD and Muse SV (2005). HyPhy: hypothesis testing using phylogenies. Bioinformatics 21: 676-679.

Porter CA, Page SL, Czelusniak J, Schneider H, et al. (1997). Phylogeny and evolution of selected primates as determined by sequences of the $\varepsilon$-globin locus and 5' flanking regions. Int. J. Primatol. 18: 261-295.

Reguigne-Arnould I, Couillin P, Mollicone R, Faure S, et al. (1995). Relative positions of two clusters of human alphaL-fucosyltransferases in 19q (FUT1-FUT2) and 19p (FUT6-FUT3-FUT5) within the microsatellite genetic map of chromosome 19. Cytogenet. Cell Genet. 71: 158-162.

Reguigne-Arnould I, Faure S, Chery M, Mota-Viera L, et al. (1996). Physical mapping of 49 microsatellite markers on chromosome 19 and correlation with the genetic linkage map. Genomics 32: 458-461.

Rutschmann F (2005). Bayesian molecular dating using PAML and multidivtime. A step-by-step manual. University of Zurich, Switzerland. http://www.plant.ch.

Sambrook J, Fritsch EF and Maniatis T (1989). Molecular Cloning - A Laboratory Manual. Cold Spring Harbor Laboratory Press, New York.

Sanger F, Nicklen S and Coulson AR (1977). DNA sequencing with chain-terminating inhibitors. Proc. Natl. Acad. Sci. U.S. A. 74: 5463-5467.

Saunier K, Barreaud JP, Eggen A, Oriol R, et al. (2001). Organization of the bovine alpha 2-fucosyltransferase gene cluster suggests that the Sec1 gene might have been shaped through a nonautonomous L1-retrotransposition event within the same locus. Mol. Biol. Evol. 18: 2083-2091.

Genetics and Molecular Research 7 (3): 663-678 (2008)

CFUNPEC-RP www.funpecrp.com.br 
Schluter D, Price TD, Mooers AU and Ludwig D (1997). Likelihood of ancestor states in adaptive radiation. Evolution 51: 1699-1711.

Schneider H (2000). The current status of the New World monkey phylogeny. An. Acad. Bras. Cienc. 72: 165-172.

Schneider H, Schneider MP, Sampaio I, Harada ML, et al. (1993). Molecular phylogeny of the New World monkeys (Platyrrhini, Primates). Mol. Phylogenet. Evol. 2: 225-242.

Schneider H, Sampaio I, Harada ML, Barroso CM, et al. (1996). Molecular phylogeny of the New World monkeys (Platyrrhini, Primates) based on two unlinked nuclear genes: IRBP intron 1 and epsilon-globin sequences. Am. J. Phys. Anthropol. 100: 153-179.

Schneider H, Canavez FC, Sampaio I, Moreira MA, et al. (2001). Can molecular data place each Neotropical monkey in its own branch? Chromosoma 109: 515-523.

Schrago CG (2007). On the time scale of New World primate diversification. Am. J. Phys. Anthropol. 132: 344-354.

Sharp PM, Averof M, Lloyd AT, Matassi G, et al. (1995). DNA sequence evolution: the sounds of silence. Philos. Trans. R. Soc. Lond. B Biol. Sci. 349: 241-247.

Sikes DS and Lewis PO (2001). PAUPRat: PAUP* implementation of the parsimony ratchet. Beta software. Version 1. University of Connecticut, Storrs.

Swofford DL (2003). PAUP*: Phylogenetic Analysis Using Parsimony (and Other Methods) Version 4 beta 10. Sinauer Associates, Sunderland.

Takahashi T, Ikeda Y, Tateishi A, Yamaguchi Y, et al. (2000). A sequence motif involved in the donor substrate binding by alpha1,6-fucosyltransferase: the role of the conserved arginine residues. Glycobiology 10: 503-510.

Thorne JL and Kishino H (2002). Divergence time and evolutionary rate estimation with multilocus data. Syst. Biol. 51: 689-702.

Thorne JL, Kishino H and Painter IS (1998). Estimating the rate of evolution of the rate of molecular evolution. Mol. Biol. Evol. 15: 1647-1657.

Xia X and Xie Z (2001). DAMBE: software package for data analysis in molecular biology and evolution. J. Hered. 92: 371-373.

Xu Z, Vo L and Macher BA (1996). Structure-function analysis of human alpha1,3-fucosyltransferase. Amino acids involved in acceptor substrate specificity. J. Biol. Chem. 271: 8818-8823.

Yang Z (1997). PAML: a program package for phylogenetic analysis by maximum likelihood. Comput. Appl. Biosci. 13: 555-556.

Zhang J, Rosenberg HF and Nei M (1998). Positive Darwinian selection after gene duplication in primate ribonuclease genes. Proc. Natl. Acad. Sci. U. S. A. 95: 3708-3713. 